\title{
ATLAS TDAQ System Integration and Commissioning
}

\author{
A. Negri*,a, on behalf of the ATLAS TDAQ collaboration** \\ ${ }^{a}$ Università degli Studi di Pavia, D.F.N.T., via Bassi 6, 27100 Pavia, Italy
}

\begin{abstract}
The ATLAS detector will be exposed to proton proton collisions at a center of mass energy of $14 \mathrm{TeV}$ with the bunch crossing rate of $40 \mathrm{MHz}$. A three-level trigger system has been designed to reduce this rate down to the level at which only interesting events are fully reconstructed. The level 1 trigger reduces the rate down to $75 \mathrm{kHz}$ via custom-built electronics. The Region of Interest Builder delivers the Region of Interest records to the second level trigger which runs the selection algorithms with the commodity processors and brings the rate further down to $\sim 3.5 \mathrm{kHz}$. Finally the Event Filter reduces the rate down to $\sim 200 \mathrm{~Hz}$ for permanent storage. We review the trigger and data acquisition architecture and its in situ commissioning using almost full detectors. Results on system functionality and performance based on the cosmic data, early experience on LHC beam in 2008 and preselected simulated events are presented.
\end{abstract}

Key words:

LHC, ATLAS, Trigger, Data Acquisition,

\section{Introduction}

ATLAS [2] is a general purpose High Energy Physics detector designed to exploit the full physics potential of the Large Hadron Collider (LHC). The latter will provide pp collisions at a center-of-mass energy of $14 \mathrm{TeV}$ with a bunch crossing rate of $40 \mathrm{MHz}$.

The Trigger and Data Acquisition system (TDAQ) of the experiment is designed to select a manageable rate of events for permanent storage and further analysis. Given a target data flux of about $300 \mathrm{MB} / \mathrm{s}$ and an expected event size of $\sim 1.6 \mathrm{MB}$, the design output rate is $\sim 200 \mathrm{~Hz}$. The required five order of magnitude on-line event reduction is achieved by three levels of trigger, characterized by a second level based on the "Region-of-Interest" (RoI) concept. Most of the system is implemented by means of commodity computing and network. Three independent Gigabit Ethernet networks connect more than 2000 multi-core x86 Linux PCs running multi-threaded $\mathrm{C}++$ applications.

\section{这 2. ATLAS TDAQ architecture}

An overview of the three levels TDAQ architecture is shown in Fig. 1. A fast first level trigger (LVL1) implemented on custom hardware is followed by two software based High Level Trigger systems (HLT): the second level trigger (LVL2) and the Event Filter (EF).

LVL1 uses coarse calorimeter and muon detector information to select events with a maximum rate of $75 \mathrm{kHz}$ and

\footnotetext{
${ }^{*}$ Corresponding author. Tel.: +39-0382-987-234

** For the full author list see [1]

Email address: Andrea.Negri@pv.infn.it (A. Negri )
}

a latency of less than $2.5 \mu \mathrm{s}$ (100 bunch crossings). During this time, the front-end electronics of the various subdetectors keep the complete event data in pipeline memory buffers. Data for rejected events are discarded, the data for selected events (up to160 GB/s) are passed via the Readout Drivers (RODs) into $\sim 1600$ Readout Buffers (ROBs). Event data remain there and are pulled by LVL2 and by the Event Builder (EB) nodes on demand. The ROBs are implemented in custom made PCI cards (ROBINs), each hosting three ROBs. The ROBINs are hosted in 150 Readout system PCs (ROSs).

For each event accepted by LVL1, a list of RoIs is given to LVL2. The list contains the positions (in $\eta, \phi$ coordinates) of all interesting objects found by LVL1 and it is assembled and communicated to LVL2 by a custom VMEbus module, the RoI Builder (RoIB). LVL2 then accesses the appropriate ROSs to pull and analyze data from the ROBs corresponding to the Regions of Interest; thus, the LVL2 uses only $2 \%$ of the full event information to take a decision and needs only $3 \mathrm{~GB} / \mathrm{s}$ to be extracted from the ROSs.

LVL2 provides a rejection factor of $20 \div 30$, bringing the rate to $3 \mathrm{kHz}$ with an average latency of $\sim 40 \mathrm{~ms}$. It is steered by dedicated supervision processes (L2SVs) in charge of receiving the RoI information from RoIB, assign the event to one of the processing units (L2PUs) and the handling of the result of the selection algorithms. These results are sent by the L2SVs to the data-flow manager (DFM), which assigns the accepted events to EB nodes (SFIs) according to load-balancing criteria. The DFM also manages the list of events that can be removed from the data-flow system, as they have either been rejected by the LVL2 or received by the EF, and periodically sends to the 


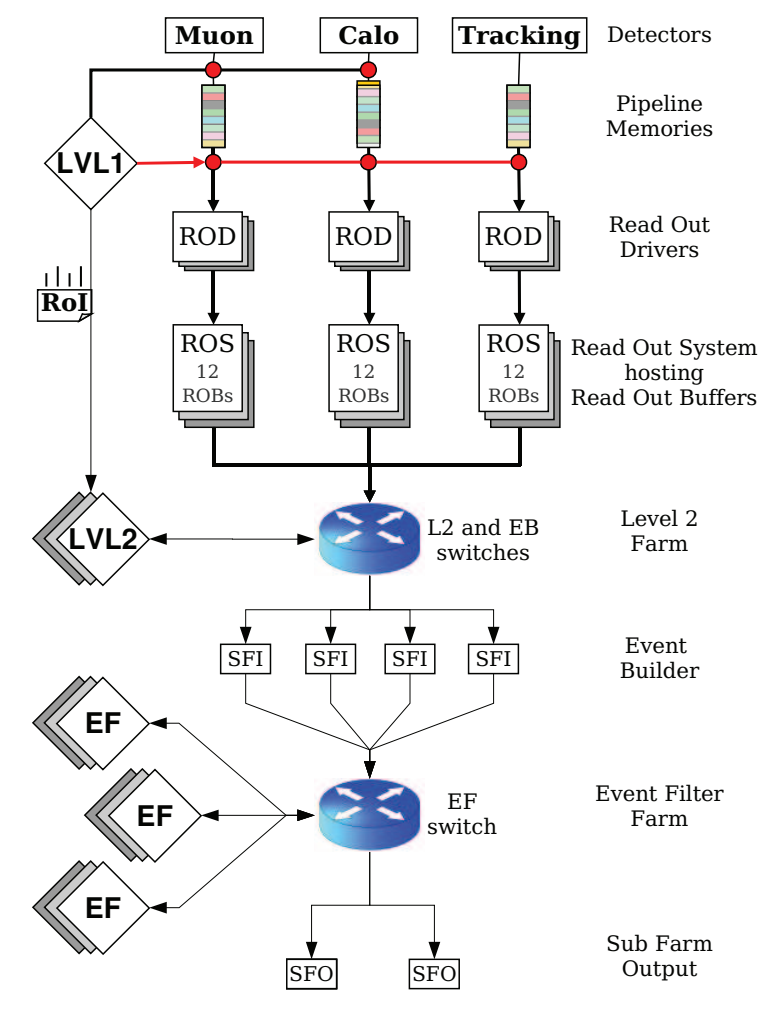

Figure 1: Layout of the ATLAS TDAQ system.

ROBs the list of data fragments to be released.

Each of the $\sim 100$ SFI applications, collect the data fragments related to any assigned event from all the ROBs and assemble them in a unique event fragment. The expected rate of events at this stage is $3.5 \mathrm{kHz}$, that corresponds to a total throughput of about $6 \mathrm{~GB} / \mathrm{s}$ out of the EB system. Upon request, the SFIs provide fully assembled events to the EF system, which analyzes the entirety of each event data to achieve a further rate reduction to $200 \mathrm{~Hz}$, with a latency of $\sim 4 \mathrm{~s}$ per event.

The EF is organized as a set of independent processor farms, connected to SFI and SFO (Sub-Farm Output) elements via the EF switch. Each EF node hosts a process in charge of data-flow functionalities (EFD) and several data processing and event selection applications, named Processing Tasks (PTs). Data security and fault tolerance are provided by the EFD, that can identify PT crashes or processing timeouts and appropriately handle the problem. Accepted events are sent for local TDAQ storage to the SFO nodes, where a data-logger application streams and indexes the events into different files, according to HLT classification. From there, the data are pulled to the central mass storage facility at CERN.

The DAQ software was written to benefit from the currently available multi-core architectures. Where possible, DAQ applications make use of multiple threads of control for increased efficiency. However, mainly for fault tolerance reasons, event selection operations in HLT are executed in multiple independent processes (L2PUs and
PTs). Those HLT applications load a common HLT event selection infrastructure.

A set of feature extraction and hypothesis making algorithms is executed one after the other to as quickly as possible reach a decision. This event processing is bootstrapped by the result of the precedent level's processing. The strategy is optimized for early rejection. See [4] for more details.

A complex on-line software infrastructure has been developed to configure, control and monitor such a large system. Details on specific aspects can be found in [5].

\section{System deployment status}

In preparation for the 2008 data-taking period most of the TDAQ sub-systems have been fully deployed. In particular networking, ROS (see [6] for a detailed report), $\mathrm{EB}$ and SFO systems were in their final configuration. The EB farm is composed of a mixture of dual-socket singlecore and dual-socket dual-core PCs, that can hosts 94 SFI applications. The required $5 \mathrm{SFO}$ nodes plus a spare have been installed and commissioned. Each node has 24 disks of 500 gigabyte, on three SATA RAID controllers, for a total of 12 terabyte per node. Considering the expected rate of accepted events and the redundancy required by the RAID protocol, the 5 SFO nodes will be able to store locally 24 hours of data, to cope with possible interruptions of the communication between ATLAS experimental area and the CERN main site.

In order to take advantage of the ever increasing hardware computation capabilities, HLT computing power will be purchase as late as possible, following the development of the ATLAS physics needs. At the moment only $\sim 40 \%$ of the final HLT farm have been installed and commissioned. Due to the deployment of two network connection, these nodes can be configured either as LVL2 or EF processors, increasing the flexibility. Even if in the last few years the CPU clock did not increase as expected, the development of the multi-core architecture allowed to achieve the expected processing power. It has been verified that, running multiple copies of L2PUs or PTs applications per node, the performance scales linearly with the number of cores. The 850 installed nodes are dual-socket quad-cores PCs with 2 GB of memory for each core.

\section{TDAQ commissioning and validation}

The ATLAS TDAQ system was successfully used during the detector commissioning and for the continuous cosmic data taking period from August to November 2008. The system was ready and functioned correctly for the first beam data taking in September 2008. Overall roughly $1 \mathrm{~PB}$ of data have been collected without major problems, confirming the functionality of the TDAQ system. In particular the SFO system has been validated at aggregate $\mathrm{I} / \mathrm{O}$ rates a factor of two larger than the design target. 


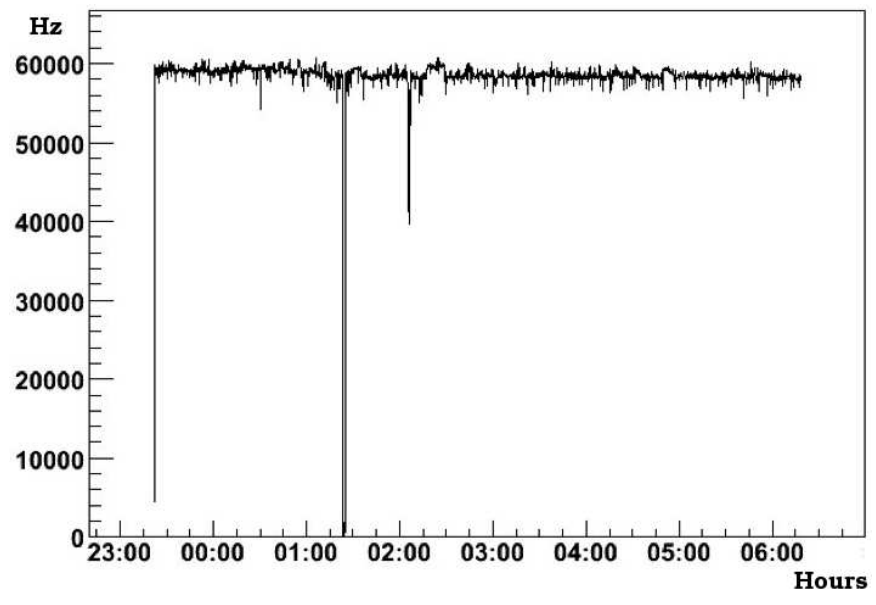

Figure 2: LVL1 trigger acceptance rate as a function of the time during $10^{31} \mathrm{~cm}^{-2} \mathrm{~s}-1$ trigger menu. The two dips are due to system wide cron jobs. This problematic behavior has been fixed since these tests.

The usage during the cosmic and first beam data-taking periods provided an invaluable feedback in terms of functionalities, stability and efficiency, however the typical system working point was quite far from its design specification. Therefore dedicated performance, robustness and scalability measurements have been performed.

For example, the stability and robustness of the full TDAQ chain was tested pre-loading simulated data into the ROS system and loading $10^{31} \mathrm{~cm}^{-2} \mathrm{~s}^{-1}$ trigger menu in the HLT nodes. The corresponding LVL1 results were seeding the LVL2 via a dedicated feeder. Even though the trigger menu was only optimized for $10 \mathrm{kHz}$, during this test a several hours of stable running condition at a LVL1 rate of $60 \mathrm{kHz}$ (Fig. 2), i.e. $80 \%$ of the target rate, was achieved. The EB was driven by the LVL2 at an aggregated bandwidth of $3 \mathrm{~GB} / \mathrm{s}$. The ROSs were able to sustain request rates up to roughly $30 \mathrm{kHz}$, while, during normal ATLAS operations, the so called "hot ROSs" are expected to work at $12 \mathrm{kHz}$.

The scalability of the system's performance to the number of ROS and SFI nodes has been tested by configuring the ROSs to internally generate data fragments with a total event size similar to the one expected in the final running conditions. Fig. 3 shows the aggregate EB throughput as a function of the number of SFI nodes, with and without sending the events to the EF system. The results show the linear scalability of the system and the almost full exploitation of the available network resources. Indeed each SFI is able to absorb as much as $114 \mathrm{MB} / \mathrm{s}$. The installed EB farm is able to sustain much more of the design $5 \mathrm{~GB} / \mathrm{s}$. Enabling the SFI output to EF system, the performance degradation is less than $8 \%$.

The performance of the communication protocol between the RoIB and the L2SVs, and hence the maximum L1 rate that can be handled, has been measured to scale linearly with the number of L2SVs. Given current mea-

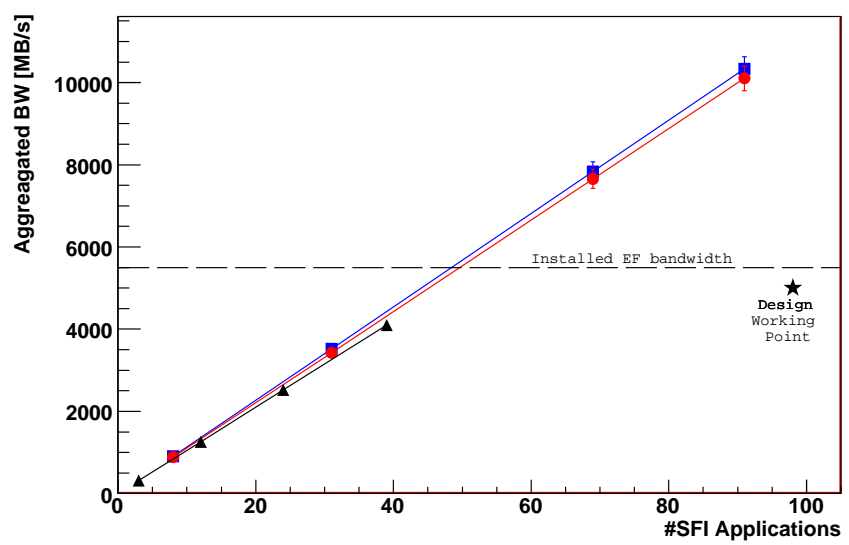

Figure 3: Aggregate EB bandwidth as a function of the number of active SFI applications using UDP/IP (squares) or TCP/IP (circles) protocol for data communication. In these measurements the events are discarded at the SFI output, while in the lowest series (triangles) the events are delivered to the EF system.

sured performance, only half of the 8 RoIB outputs will be used, i.e.: no more than $4 \mathrm{~L} 2 \mathrm{SVs}$ will be required to sustain the full LVL1 rate.

\section{Conclusions}

Most of the TDAQ system has been installed and commissioned. In particular the read-out and the EB systems are fully deployed. The completion of the procurement and installation of the HLT processing nodes is scheduled to match the LHC schedule and the plans for ATLAS data taking. The TDAQ system is regularly and successfully used for the ATLAS commissioning and data-taking operations. Moreover, dedicated TDAQ performance tests are periodically carried-out in order to integrate the datataking results. Coherent software releases containing both the data-flow applications and the infrastructure components are produced several times per year.

Based on the current achievements, the TDAQ system is robust and scalable enough to fulfill the ATLAS requirements.

\section{References}

[1] ATLAS TDAQ Collaboration, The ATLAS Trigger/DAQ Authorlist, version 1.0, ATL-DAQ-PUB-2008-004, CERN, Geneva, 2008, http://cdsweb.cern.ch/record/1141516

[2] ATLAS Collaboration, The ATLAS experiment at the CERN Large Hadron Collider, JINST 3 S08003, 2008

[3] ATLAS Collaboration, ATLAS, High-Level Triggers, Data Acquisition and Controls, CERN/LHCC/2003-0022, Geneva, CERN, 2003

[4] ATLAS Collaboration, Commissioning of the ATLAS high-level trigger with single beam and cosmic rays, these proceedings.

[5] ATLAS TDAQ Collaboration, Configuration \& control of the ATLAS trigger and data acquisition, these proceedings.

[6] M. Joos et al, The ATLAS ReadOut System - performance with first data and perspective for the future, these proceedings. 